ISSN: $1679-3013$

\title{
DISTRIBUTION OF THE MESOZOOPLANKTON AT CONTINENTAL SHELF OFF SERGIPE, BRAZIL.
}

\author{
Hortência Maria Pereira ARAUJO ${ }^{1}$ \\ Viviane Andrade RIBEIRO ${ }^{2}$
}

Recebido em:

Aceito em: $16 / 03 / 2006$

\begin{abstract}
The purpose of this work was to study the composition and abundance of the mesozooplankton at two stations at the continental shelf off Sergipe. At each station, oblique plankton tows were carried using plankton nets with $200 \mu \mathrm{m}$ mesh size, in six hours intervals during three consecutive days. One hundred and three taxa were identified and Copepoda was the dominant group with fifty one species. The mesozooplankton assemblage was composed by coastal, neritic and oceanic tropical species. There were significant differences in composition and abundance between the two studied stations $(p<0,05)$ but not between tidal stages. At station 1 , the most abundant species were Creseis acicula, Oikopleura spp., Temora stylifera, Paracalanus quasimodo and Aglaura hemistoma, and at station 2 Paracalanus quasimodo, Paracalanus spp., Siphonophora, Gastropoda and Decapoda larvae. Coastal species such as Parvocalanus crassirostris, Acartia lilljeborgi, Euterpina acutifrons and Corycaeus giesbrechti occurred only at the last station, located close to the mouth of the São Francisco River, where surface salinity and transparency values were low. In this station the salinity average value at surface was $30,8 \pm 1,9$ and at the bottom layer was

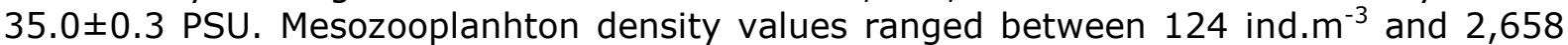
ind. $\mathrm{m}^{-3}$ at station 1 , and from 734 ind. $\mathrm{m}^{-3}$ to 3,061 ind. $\mathrm{m}^{-3}$ at station 2. A Monte Carlo test based on a canonical ordination revelead that local depth and water transparency were significantly correlated to the mesozooplankton distribution.
\end{abstract}

Key words: zooplankton community, Copepoda, shelf off Sergipe, tropical.

\section{DISTRIBUIÇÃO DO MESOZOOPLÂNCTON NA PLATAFORMA CONTINENTAL DE SERGIPE, BRASIL}

\section{RESUMO}

A proposta desse trabalho foi estudar a composição e abundância do mesozooplâncton em duas estações, na plataforma continental de Sergipe. Em cada estação, arrastos oblíquos de plâncton foram feitos utilizando-se uma rede de plâncton de $200 \mu \mathrm{m}$ de abertura de malha, em intervalos de seis horas, durante três dias consecutivos. Cento e três táxons foram identificados e Copepoda foi o grupo dominante com cinqüenta e uma espécies. A assembléia mesozooplanctônica foi composta por espécies tropicais costeiras, neríticas e oceânicas. Houve diferença significativa na composição e abundância entre as duas estações estudadas $(p<0,05)$, mas não entre os estágios tidais. Na estação 1 as espécies mais abundantes foram Creseis acicula, Oikopleura spp., Temora stylifera, Paracalanus quasimodo e Aglaura hemistoma e na estação 2, Paracalanus quasimodo, Paracalanus spp., Siphonophora e larvas de Gastropoda e Decapoda. Espécies costeiras tais como Parvocalanus crassirostris, Acartia lilljeborgi, Euterpina acutifrons e Corycaeus giesbrechti ocorreram apenas na estação 2, que está localizada próxima à foz do Rio São Francisco, onde os valores de salinidade de superfície e a transparência da água foram baixos. Nesta estação o valor médio de salinidade na superfície foi $30,8 \pm 1,9$ e na camada de fundo foi $35.0 \pm 0.3$ PSU. Valores de densidade flutuaram entre 124 ind. $\mathrm{m}^{-3}$ e 2.658 ind. $\mathrm{m}^{-3}$ na estação 1 e de 734 ind. $\mathrm{m}^{-3}$ a 3.061 ind. $^{-3}$ na estação 2. O teste de Monte Carlo, baseado em uma ordenação

Contatos: Laboratório de Zooplâncton, Departamento de Biologia, Universidade Federal de Sergipe

${ }^{1}$ E-mail: haraujo@ufs.br; bioemail@hotmail.com 
ARAUJO; RIBEIRO. Distribution of the Mesozooplankton at continental shelf off Sergipe, Brazil.

canônica, revelou que a profundidade local e transparência da água estiveram correlacionadas significativamente com a distribuição do mesozooplâncton.

Palavras-chave: Comunidades zooplanctônicas, Copepoda, plataforma de Sergipe, tropical.

\section{INTRODUCTION}

Zooplankton community plays an important role in the biological cycle of carbon and other elements in the ocean (PALOMRES-GARCIA; GÓMEZ-GUTIERREZ, 1996) and presents variable distribution patterns and indicative species of the environmental conditions (PARANAGUÁ et al., 2004).

Variations in composition and abundance of the marine zooplankton are related to water mass movements (DESSIER and DONGUY, 1985; KOUWENBERG, 1994), available food (PINCA and DALLOT, 1997; RIOS-JARA, 1998), biological interaction such as predator-prey association (WIAFE and FRID, 1996), bottom topography (ARASHKEVICH et al., 2002) or combined effects of climatology, hydrological variables and biotic factors (RIOS-JARA, 1998). Schwamborn et al. (1999) added the exportation of organisms from estuaries to continental shelf waters.

The continental shelf off Sergipe is located in the northeastern region of Brazil, where the tropical climate is characterized by high temperatures all over the year, and it is well defined by two seasons: dry season, from September to February, and rainy season, from March to August. Five rivers discharge their waters in this area (São Francisco, Japaratuba, Sergipe, Vaza-Barris and Piauí-Real). The most important one is the São Francisco. This river has a length of $3,161 \mathrm{~km}$ and an average flux of $2,850 \mathrm{~m}^{3} \cdot \mathrm{s}^{-}$ ${ }^{1}$ (CUNHA and GUERRA, 2003). The turbid waters of the São Francisco plume are moved toward the south by coastal currents and inhibit the development of reef constructions in the area under its influence (VILLWOCK, 1994; COUTINHO, 1995).

The shelf off Sergipe is narrow with an average width of $30 \mathrm{~km}$, and an abrupt slope interrupted by two large submarine canyons of the São Francisco and the Japaratuba Rivers and by several narrow shallow channels that reach up to $10 \mathrm{~km}$ from the shore line (FRANÇA et al, 1976; SUMMERHAYES; et al., 1976). In consequence of these erosive characteristics the width of the shelf ranges from 12 to $35 \mathrm{~km}$, and the 50 $\mathrm{m}$ isobath is close to the shelf break so that almost all shelf can be considered as a continental inner shelf (CUNHA and GUERRA, op. cit.).

The knowledge about the biological community dynamics is necessary in the area to evaluate the natural environmental dynamics and the anthropic action such as fishing activities and oil and natural gas production. The distribution patterns of the zooplankton in this shelf area are still unknown. The purpose of this work is to study the composition and abundance of the mesozooplankton community at two stations in the continental shelf off Sergipe, so as to contribute towards the knowledge of its distribution patterns.

\section{MATERIAL AND METHODS}

\section{Sampling and sample processing}

Sampling was carried out at two stations in December 2002, during the dry season of the year. Station 1 is located 500 meters from an oil production platform and close to the Japaratuba submarine canyon ( $\left.10^{\circ} 59^{\prime} \mathrm{S} ; 36^{\circ} 56^{\prime} \mathrm{W}\right)$ where the local depth is 27 meters, while station 2 is located in the area influenced by the São Francisco River $\left(10^{\circ}\right.$ $37^{\prime} \mathrm{S} ; 360^{\prime} \mathrm{W}$ ) and close to the São Francisco submarine canyon. The local depth at this station is 20 meters (Fig. 1).

Water was sampled with a Van Dorn bottle at two levels of the water column: below the surface and near the bottom. The following variables were measured: transparency (Secchi disc); temperature (digital thermometer); salinity (digital conductivity meter); dissolved oxygen and chlorophyll-a (STRICKLAND; PARSONS, 1965) and nutrients ammonia, nitrite, nitrate, phosphate and silicate (APHA, 1995). 


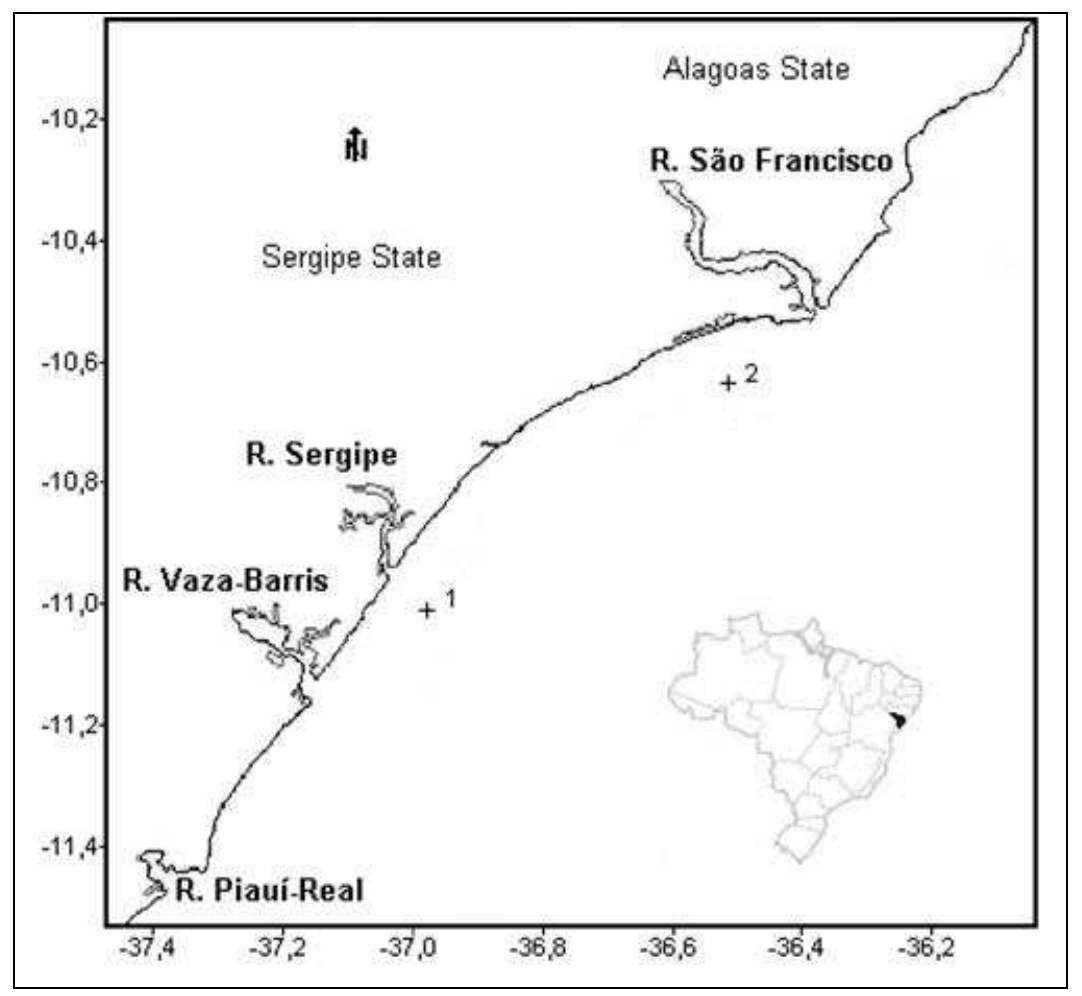

Figure 1. Continental shelf off Sergipe with the sampling stations (stations 1 and 2).

At each station, an oblique plankton tow was carried out every six hours, for three consecutive days, using plankton nets with $200 \mu \mathrm{m}$ mesh size. A General Oceanic flowmeter was adjusted in the net mouth for measuring the filtered water volume. Samples were preserved in a buffered $4 \%$ formaldehyde solution.

Zooplankton abundance was estimated from sub-samples drawn with a $5 \mathrm{ml}$ Stempel pipette and counted under a dissecting microscope. Mesozooplankton organisms were identified, most of them down to species or genus levels. The abundance was expressed as number of individuals per cubic meter (ind. $\mathrm{m}^{-3}$ ).

\section{Data analyses}

Diversity of the mesozooplankton assemblage was expressed by Shannon-Wienner index (SHANNON-WIENNER, 1948) and equitability by Pielou's eveness index (PIELOU, 1977). A one-way ANOVA was used to test differences in zooplankton abundance between hauls in each station (hauls, night and day). The variance homogeneity was tested through the Levene test. The non-parametric Mann-Whitney $U$ test $(\alpha=0,05)$ was used to determine whether the zooplankton densities were different in the two sampled stations and at night and day (ZAR, 1984). The analyses were conducted employing the computer package STATISTICA 5.1 (StatSoft Inc.).

In order to classify the samples and the taxa, a cluster analysis was applied to the density matrix, after the data had been transformed by $\log (x+1)$. The Bray-Curtis dissimilarity coefficient was used and taxa with density less than 5,0 ind. $\mathrm{m}^{-3}$ were eliminated. The samples were represented by two digits: the first one corresponding to station 1 or 2 and the last one to the tidal stage ( $E$ : ebb tide and F: flood tide).

Associations between mesozooplankton communities and environmental variables were analyzed using canonical correspondence analysis - CCA through the CANOCO program (TER BRAAK, 1987; 1989). The robustness of this analysis was determined using the Monte Carlo's permutation test (TER BRAAK, 1986). 
ARAUJO; RIBEIRO. Distribution of the Mesozooplankton at continental shelf off Sergipe, Brazil.

\section{RESULTS}

\section{Hydrologic variables}

Mean water temperatures at sampling stations were high and bottom values were higher especially at station 2 where the difference between surface and bottom reached $4^{\circ} \mathrm{C}$. Transparency $(2.4 \pm 0.7 \mathrm{~m})$, surface salinity $(30.8 \pm 1.9 \mathrm{PSU})$ and $\mathrm{pH}(8.3)$ values were significatively lower at station 2, indicating the influence of the São Francisco flow in this area (Tab. 1). Nutrient $\left(\mathrm{NH}_{4}, \mathrm{NO}_{2}, \mathrm{NO}_{3}, \mathrm{PO}_{4}\right.$ and $\left.\mathrm{Si}(\mathrm{OH})_{4}\right)$ concentrations showed a high variability. Minimum, maximum, mean and standard deviation values were in the Table 1.

Table 1 - Minimum and maximum values, mean and standard deviation of the hydrologic variables at surface (S) and bottom (B) of the Sergipe shelf in December 2002.

\begin{tabular}{|c|c|c|c|c|c|c|c|c|}
\hline \multirow[b]{2}{*}{ Variabes } & \multicolumn{4}{|c|}{ Strtion 1} & \multicolumn{4}{|c|}{ Strtion 2} \\
\hline & 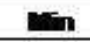 & ㅂ. & ven & sn & in & max & then & s] \\
\hline Temparalue ("C) (S) & 26.0 & 27.0 & 26.7 & 0.5 & 250 & 200 & 26.3 & 1.1 \\
\hline Tempardate $\left({ }^{(E)}(B)^{*}\right.$ & 250 & 26.0 & 252 & 0.4 & 24.0 & 250 & 24.1 & 0.3 \\
\hline pH $(\boldsymbol{S})^{*}$ & 8.3 & 8.4 & 8.4 & 0.0 & 82 & $\mathbf{8 . 3}$ & 8.3 & 0.0 \\
\hline pH B) ( $^{*}$ & $\mathbf{8 . 3}$ & 8.4 & $\mathbf{8 . 4}$ & 0.0 & 82 & $\mathbf{8 . 3}$ & $\mathbf{8 . 3}$ & 0.0 \\
\hline Satiry (S) (PSU) ${ }^{*}$ & 327 & 342 & 33.7 & 0.5 & 27.0 & 33.6 & 30.8 & 1.9 \\
\hline Sality (B) (PSI) & 34.7 & 352 & 34.9 & 0.1 & 34.6 & 35.4 & 350 & 0.3 \\
\hline Tranparemsy $(\varpi)^{*}$ & 8.0 & 11.0 & 92 & 1.3 & 1.7 & 30 & 24 & 0.7 \\
\hline Dissolved Oxgea (S) (mgl' & 6.7 & 8.3 & 7.4 & 0.5 & 7.0 & 8.9 & 8.0 & 0.7 \\
\hline Dissohed Orgen (B) (agl-1) & 6.9 & 82 & 7.4 & 0.4 & 7.0 & 90 & 7.8 & 0.7 \\
\hline Ammonia (S) $\cap g I^{-1}$ ) & 24 & 89.7 & 23.6 & 31.6 & 3.8 & 6.8 & 5.7 & 0.8 \\
\hline$A$ Amonia (B) $\left(\mathrm{g}^{-1} \mathrm{I}^{\mathrm{yt}}\right.$ & 29 & 109.8 & 325 & 41.7 & 4.4 & 9.8 & 6.5 & 1.3 \\
\hline Minte (S) $\left(\mathrm{Fgl}^{-1}\right)^{*}$ & 0.1 & 0.6 & 0.3 & 0.2 & 0.1 & 1.5 & 0.8 & 0.4 \\
\hline Alite (B) $\left(\mathrm{F} g \mathrm{I}^{-1} \mathrm{y}^{ \pm}\right.$ & 0.1 & 0.7 & 0.3 & 0.2 & 0.1 & 12 & 0.6 & 0.3 \\
\hline Whate (S) $\left.\rightarrow g L^{-1}\right)^{*}$ & 0.7 & 8.5 & 5.1 & 29 & 8.0 & 14.1 & 10.9 & 22 \\
\hline Hithe(S) (aI-1) * & e.7 & 12.8 & 52 & 4.1 & $\mathbf{8 . 5}$ & 20.9 & 11.5 & 3.3 \\
\hline Ploosple (S) $\left(\mathrm{ggl}^{-1}\right)$ & 0.6 & 8.4 & 1.9 & 25 & 0.6 & 8.4 & 1.7 & 23 \\
\hline Plosplute (B) (1ngl & 0.6 & 8.4 & 1.7 & 26 & 0.6 & 7.1 & 20 & 21 \\
\hline Sirale (S) $\left(\mathrm{Rg}^{-1}\right)$ & 129.7 & 954.9 & 658.7 & 304.0 & 570.6 & 1375.8 & 879.8 & 231.5 \\
\hline Sticale B) ( $\left.\mathrm{Bg}^{-1}\right)$ & 175.1 & 483.4 & 2065 & 1028 & 193.4 & 450.9 & 2728 & 79.7 \\
\hline Chlophes $\left(\operatorname{Rgl}^{-1}\right)$ & 0.1 & 0.6 & 0.1 & 0.2 & 0.1 & 0.3 & 0.1 & 0.1 \\
\hline
\end{tabular}

Significative difference $(p<0.01) *(p<0.05) * *$ between stations 1 and 2 .

\section{Species composition and distribution}

One hundred and three taxa were identified. Copepoda was the dominant group with fifty one species. Decapod larvae were the most abundant meroplanktonic organisms.

At station 1 Copepoda formed $40.9 \%$ of the mesozooplanktonic community and Temora stylifera (8.23\%), Paracalanus quasimodo $(7.45 \%), \quad$. indicus $(5.05 \%)$, Clausocalanus furcatus (4.17\%) and Euchaeta marina $(2.95 \%)$ were the most abundant species. Creseis acicula (14.64\%), Appendicularia (12.76\%) and Hydromedusae $(6.66 \%)$ were also important taxa. Meroplanktonic larvae composed $4.64 \%$ of the community (Tab. 2; Fig. 2). The mesozooplankton average density was $587 \pm 692$ ind. $\mathrm{m}^{-3}$. The density values ranged from 124 ind. $\mathrm{m}^{-3}$ ( $09 \mathrm{AM}$, ebb tide) to 2,658 ind. $\mathrm{m}^{-3}$ (03 PM, flood tide) (Fig. 3). Creseis acicula (650 ind $\mathrm{m}^{-3}$ ) and Aglaura hemistoma (330 ind. $\mathrm{m}^{-3}$ ) were the most abundant species in the flood tide.

At station 2, Copepoda reached $61.4 \%$ of the mesozooplankton assemblage (Fig. 2). The most important species were Paracalanus quasimodo (26.12\%), Temora stylifera (4.93\%), Paracalanus aculeatus (3.32\%), Corycaeus giesbrechti $(2.89 \%)$ and Oithona plumifera (2.78\%). 
Meroplanktonic larvae comprised $13.5 \%$ of the community (Tab. 2 ). The average density of the mesozooplankton was $1,678 \pm 765$ ind. $\mathrm{m}^{-3}$ and density values ranged between 734 ind. $\mathrm{m}^{-3}$ (00 AM, flood tide) and 3,061 ind. $\mathrm{m}^{-3}$ (12 PM, flood tide) (Fig. 3). In the flood tide, the most abundant taxa were Gastropoda larvae (524 ind. $\mathrm{m}^{-3}$ ), Paracalanus quasimodo (512 ind. $\mathrm{m}^{-3}$ ) and Decapoda larvae (305 ind. $\mathrm{m}^{-3}$ ).

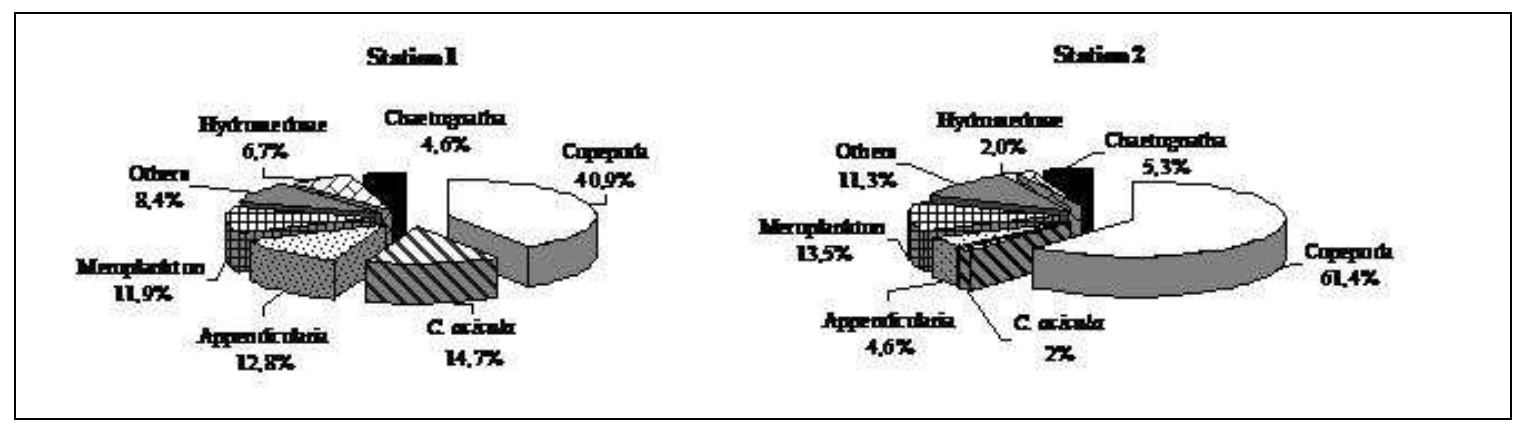

Figure 2 - Relative abundance of the mesozooplanktonic taxa of the Sergipe shelf in December 2002.

Table 2 - Relative abundance (\%), average density (ind. $\mathrm{m}^{-3}$ ) and standard deviations of mesozooplanktonic taxa of the Sergipe shelf (stations 1 and 2) in December 2002.

\begin{tabular}{|c|c|c|c|c|c|c|c|c|c|c|c|c|c|}
\hline \multirow{3}{*}{ 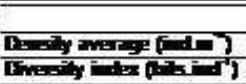 } & \multicolumn{4}{|c|}{ \%-fiom 1} & \multirow{2}{*}{\multicolumn{2}{|c|}{$\frac{5 y \operatorname{tion} 2}{1 \mathrm{fr} 2 \mathrm{C}}$}} & \multirow[b]{2}{*}{$T=0$} & \multicolumn{4}{|c|}{ 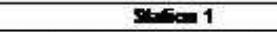 } & \multicolumn{2}{|c|}{ Dhtion 2} \\
\hline & & & 52 & & & & & $\overline{\mathbf{E}}$ & ME & 표 & $\overline{\mathbf{E}}$ & Me & F \\
\hline & & & 45 & & & 161 & Citheng accilats & - & - & - & DDA & 1132 & $\mathbf{1 1 7}$ \\
\hline EYE $=8$ & & & 0.76 & & & 0.71 & 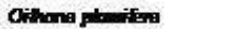 & 1 BA & 12: & 30म & $2 \pi$ & B214 & $\pi 7$ \\
\hline Trom & $\overline{\mathbf{E}}$ & $\mathrm{He}$ & $\mathbf{g}$ & $\bar{\square}$ & 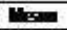 & $\overline{\mathbf{g}}$ & Othans moxd & - & - & - & D.) & 11.54 & 11.12 \\
\hline Rafinilaria & 250 & 2ZO & E.13 & D30 & 20.38 & $\overline{24.19}$ & Oithma s.higera & - & - & - & 0.32 & $\mathbf{8 . 1 2}$ & 7 Hig \\
\hline Fur:miniferida & D.14 & 209 & 150 & DDE & 410 & $3 \mathbf{5 0}$ & Oilhong beis & D.07 & 237 & D74 & DEH & 13.73 & 1DED \\
\hline Findexis qp & - & - & - & DD2 & 48: & - & Oritheng App & D. $\pi$ & 13.8. & 2244 & D.9A & 18.9 & 15.59 \\
\hline 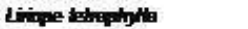 & D.02 & 1.52 & - & Dos & 238 & 280 & Happorfuride & - & - & - & 0.03 & 1.81 & D.5 \\
\hline 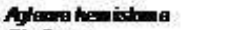 & 5.7A & 3402 & $\mathbf{9 3 3 7}$ & D.74 & 13 BA & 891 & 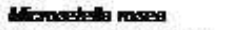 & - & - & - & DD1 & 1.38 & - \\
\hline Chesa op & - & & - & DD1 & 1,388 & - & Henigrips thatasies & - & - & - & DD1 & 1.85 & - \\
\hline Hydmometreas & D.85 & 0.02 & 1039 & 1.17 & 19E- & 18. & 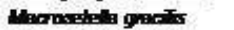 & D.D1 & $\mathrm{D} \pi$ & - & - & - & - \\
\hline Sphuruphur & 189 & 1210 & 1887 & Ex & 113.02 & 81.18 & 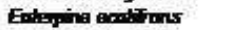 & - & - & - & DDA & 280 & 1.13 \\
\hline Oilhis & D.13 & 9.5 & - & DDS & 296 & $2 \pi 2$ & Oncrea media & 1.109 & $7 \mathrm{FB}$ & 884 & 108 & 19.80 & 18.80 \\
\hline Clemphing & - & - & - & DDe & $3 \mathrm{FB}$ & - & Oncaea werwath & D.D9 & 30 A & $\mathbf{3 . 1 5}$ & D.20 & 5.5 & 253 \\
\hline Pulychich (harke) & D.39 & 213 & 281 & DE & $5: 5$ & 102 & Onraed hp & 0.27 & $\mathbf{3 . 1 7}$ & 137 & 0.28 & 7.98 & 12月: \\
\hline Pulydhacka (aih) & D.89 & Zas & 37 HD & - & - & - & 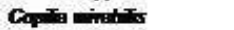 & - & - & - & D.DA & 29 & 1.8 \\
\hline 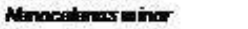 & D.10 & $1 \mathrm{FBg}$ & 138 & DD1 & 28 & - & 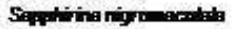 & D.05 & 15 & 1F2 & - & - & - \\
\hline 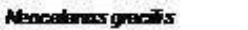 & D.D1 & D.74 & - & - & - & - & Sephede ap & 0.05 & 335 & - & - & - & - \\
\hline Ihalinda rolyins & D. 8 & 13:0 & 531 & D.05 & $2 \mathrm{FB}$ & D.59 & Caymens perinsw & DZZ & 201 & 3.44 & D.18 & 212 & D. Bf \\
\hline Cotaintae & D.D7 & 1.24 & D.47 & DD3 & 5.52 & - & 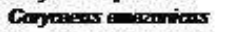 & - & - & - & $\mathbf{D . 5 3}$ & 15.18 & 13. $\mathrm{H}$ \\
\hline Arnopitinestangineris & D.09 & 218 & D.57 & - & - & - & Cangraeus gistredti & DEA & HA4 & 5:90 & 289 & 80 & 3230 \\
\hline Chestinespeng & D.18 & 12.97 & - & DD2 & 102 & - & Congrieus q7p & D. 8 & 221 & 2E- & D.5 & 9.99 & 7. \\
\hline Cahertines qpp & 1.14 & 10.03 & $12 \mathrm{EA}$ & D.41 & $\mathbf{8 3 1}$ & 7.32 & Faminde gracs & 1.188 & 7F1 & 13.95 & D.DB & 405 & 15 \\
\hline Plenatanes quasiento & 7.6 & ent & 71.37 & Zi12 & AR:Z & 32156 & 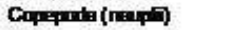 & D.TA & $5 \mathrm{HS}$ & 7EG & 1.78 & 2701 & 27 \\
\hline 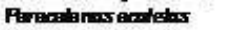 & DES & HET & $10 \mathrm{Do}$ & 3.32 & Bos & 118.98 & Oaflisonda & D. 8 & 483 & 121 & 1.) & Eid & 2272 \\
\hline Paranianes indires & 5.D5 & 28H & $37 \mathrm{FB}$ & 106 & 3a_t7 & 252 & Cimipedie (naplin) & D.07 & 1 Fit & DES & D.DB & $3 \pi$ & 1Fi \\
\hline Peranghnes cqp & 1.38 & 1218 & 19.97 & 5.9 & 9217 & 8372 & Cinipala (cpili) & D.12 & 28: & 1.82 & D.DA & 3.74 & 1.58 \\
\hline Farnocatanes massingstis & - & - & - & D2Z & 298 & $5 . \pi$ & 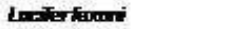 & 0.22 & $\mathbf{3 . 1 5}$ & 152 & 1.32 & 207 & z.99 \\
\hline Sherarithes masses & D.D1 & D.9A & - & - & - & - & Shmilipaita (lareng) & DDA & D87 & DSA & - & - & - \\
\hline Sheoratines pates & - & - & - & DABg & 15.31 & 20.39 & Dexpipita (haree) & 3.79 & 248 & 23.19 & $\mathbf{5 . 1 3}$ & : & 7407 \\
\hline 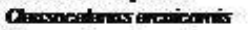 & - & - & - & DDZ & 402 & - & Wyitaxer & D.D3 & DE8 & DDa & - & - & - \\
\hline 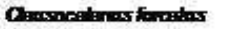 & 217 & 24.8 & 37.34 & D28 & B24 & 1139 & leqpinta & D.13 & 180 & DAD & D.D5 & 251 & D. 2 \\
\hline Chernofitimes opp & D.02 & $1 \mathrm{HI}$ & - & DDP & 2) & D.92 & Amptipina & 0.31 & 435 & 3 ल & D.M & 1250 & 1234 \\
\hline Exhacta maing & 256 & E.97 & 2400 & D.eB & 9E2 & 1D. 72 & 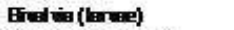 & 0.32 & 252 & 1Fi & D.89 & 14.19 & 11. ㅐㅐ \\
\hline Larimdis antis & D.D1 & D.84 & - & - & - & - & Gazhupita (lervad) & 3.71 & 21.78 & $\mathbf{z}$ 田 & 5.05 & $\mathbf{8 L} \pi$ & 14132 \\
\hline Fhaema spinger & - & - & - & DD1 & 28 & - & Chespis acinim & 1484 & 9381 & 185.15 & 1.82 & ena & 7.28 \\
\hline H:hpthes anoconates & - & - & - & DD1 & 288 & - & Pulpupida & $1 \mathrm{H1}$ & 1419 & 2184 & D. $\pi$ & 1404 & 11_日A \\
\hline 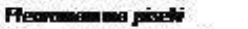 & - & - & - & DD1 & 288 & - & 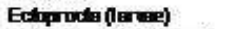 & 1.50 & 10.94 & 9角 & 109 & 19..88 & 18.98 \\
\hline Coltrpages hinginenis & - & - & - & DD1 & 287 & - & 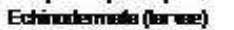 & 0.52 & 5.78 & 1039 & D.44 & 8.14 & 8.32 \\
\hline Centripages firatas & $D .23$ & 274 & 202 & 1.14 & 201 & $21 . \pi$ & Plemong ita dhaces & D.D1 & D.98 & - & - & - & - \\
\hline$F=0$ and & $\mathbf{8 z 3}$ & 832 & 292 & 493 & 8273 & 73.14 & Sagth hisidh & 0.78 & $8 \mathrm{ng}$ & $8 \pi$ & D.20 & Hep & 3.D4 \\
\hline 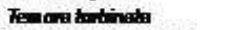 & & - & - & D.83 & 2 FE & D.32 & Sagin this & 0.89 & 128 & 792 & 1. 7 & 20.34 & Z10 \\
\hline Cindaria pachyolachla & D.PA & 304 & - & DD1 & $28=8$ & - & Sagith endida & 1.94 & 13霖 & 1378 & 1.19 & 2002 & 15.19 \\
\hline Cindaria ipp. & - & - & - & DD1 & 1.B7 & - & 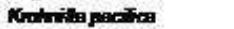 & DEDO & 500 & 121 & 107 & 17.56 & 1481 \\
\hline 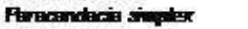 & D.D2 & $1 \mathrm{FBg}$ & - & - & - & - & Saghte ip & D.52 & 491 & 5.97 & 1.07 & 17.98 & $13 \mathrm{Et}$ \\
\hline 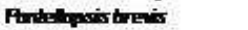 & D.DB & 1.41 & 1 DA & DD2 & $3 \mathbf{A 4}$ & - & Anpoteasidion & D.DB & 103 & D.41 & D.38 & 7.田 & 214 \\
\hline 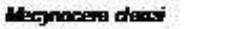 & D. 8 & 7.51 & $13 \pi$ & 1 He & $30 \mathrm{Hi}$ & 30.37 & 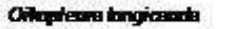 & 1 IEs & 10.5 & 17.52 & $12 \pi$ & al. 7 & 13.31 \\
\hline 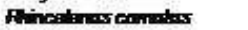 & D.D1 & D\&A & - & - & - & - & Ollypeng ppe & 10.41 & B1.14 & BARR & 302 & 50.74 & Dnio \\
\hline 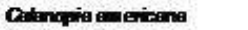 & D.8 & $10 \mathrm{ng}$ & 851 & 291 & a.74 & 4.98 & Fontinia pp & DER & 423 & 20 & D.01 & 1.84 & - \\
\hline 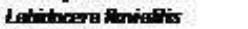 & D.DA & D.98 & D20 & DD2 & 1.72 & D.14 & 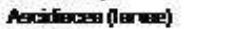 & D.D1 & $\mathrm{D} n$ & - & - & - & - \\
\hline Letimbrers qpp & 0.02 & 1 . F 8 & - & DD1 & 287 & - & Silping & D_-6 & H.4 & 7.18 & D.05 & 5.41 & 451 \\
\hline 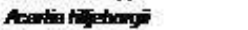 & & - & - & DD2 & 48 & - & Dofinide & D.S & HDS & 1001 & D.DB & 8.53 & $1.7 \mathrm{G}$ \\
\hline Anesis danae & D.12 & 287 & 1.15 & DD1 & 287 & - & 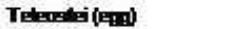 & D.31 & 207 & 133 & D.20 & 5.11 & $32 \pi$ \\
\hline Q Almane nina & D.D8 & 1.91 & 1.10 & D21 & 5.32 & 328 & Thereitei (larea) & D.12 & 170 & 1.18 & 0.32 & 5.77 & 1.56 \\
\hline
\end{tabular}


ARAUJO; RIBEIRO. Distribution of the Mesozooplankton at continental shelf off Sergipe, Brazil.

The average diversity values were high (4.79 bits.ind ${ }^{-1}$ - station 1 and 4.61 bits.ind $^{-}$ 1 - station 2) (Tab. 1). At station 1 , the lowest value (2.94 bits.ind $^{-1}$ ) was registered at 07 AM during the ebb tide when only 23 taxa were registered, and Creseis acicula formed $37.2 \%$ of the mesozooplankton assemblage. The evenness was 0.65 . The highest value was 4.83 bits.ind $^{-1}$ at 03 AM during the flood tide when 61 taxa were identified and the evenness value was 0.82 . At this station eighty one taxa were identified. At station 2 , minimum diversity was 2.80 (13 PM, flood tide), when only 24 taxa were registered and the equitability was low (0.52) due to the dominance of Paracalanus quasimodo that formed $55 \%$ of the community. Maximum diversity was 4.78 bits.ind $^{-1}$ at 00 AM during flood tide, when the evenness reached the maximum value $(0.86)$ and eighty nine taxa were identified in this sample (Fig. 3).

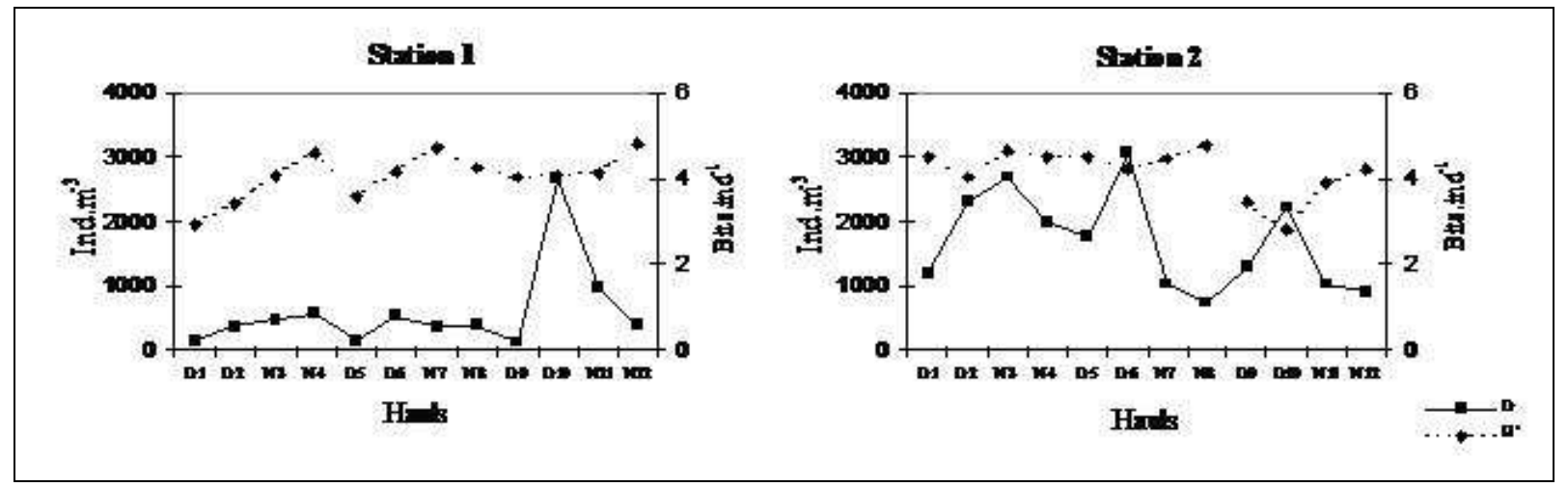

Figure 3 - Density (D) and diversity index $\left(\mathrm{H}^{\prime}\right)$ of the mesozooplankton of the Sergipe shelf during diurnal (D) and nocturnal (N) hauls in December 2002.

The ANOVA revealed no significant temporal variations of zooplankton densities. However, the Mann-Whitney $U$ test showed that zooplankton densities were significantly lower at station 1 than at station 2 (Fig. 4) and that there is no significative defference between densities of the mesozooplankton from night and daily hauls at both stations $(p>0.05)$.

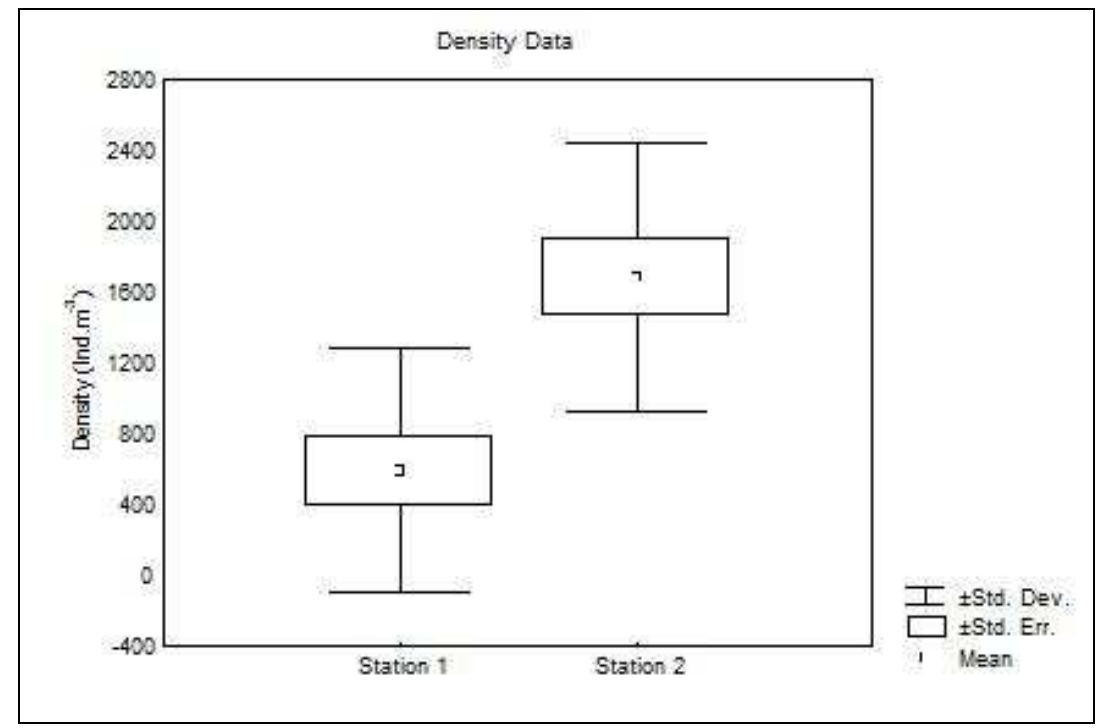

Figure 4 - Mann-Whitney $U$ test. Mean density, standard deviation and standard error of the mesozooplankton of the Sergipe shelf (stations 1 and 2) in December 2002.

The cluster analysis revealed that the mesozooplankton distribution presented a strong spatial variation with two distinct sample groups. The first one comprised those samples from station 2, where the mesozooplankton density values were higher, and the second group comprised those hauls from station 1 located in a deeper area close to an oil production platform, with waters more transparent (Fig. 5). 
The taxa cluster analysis presented three groups (Tab. 3; Fig. 6). Group 1 was composed by abundant and frequent species of both stations. Group 2 was mostly composed by exclusive species of station 1 or taxa that were more abundant in this station. And group 3 was composed by taxa that occurred exclusively or in higher abundances at station 2 .

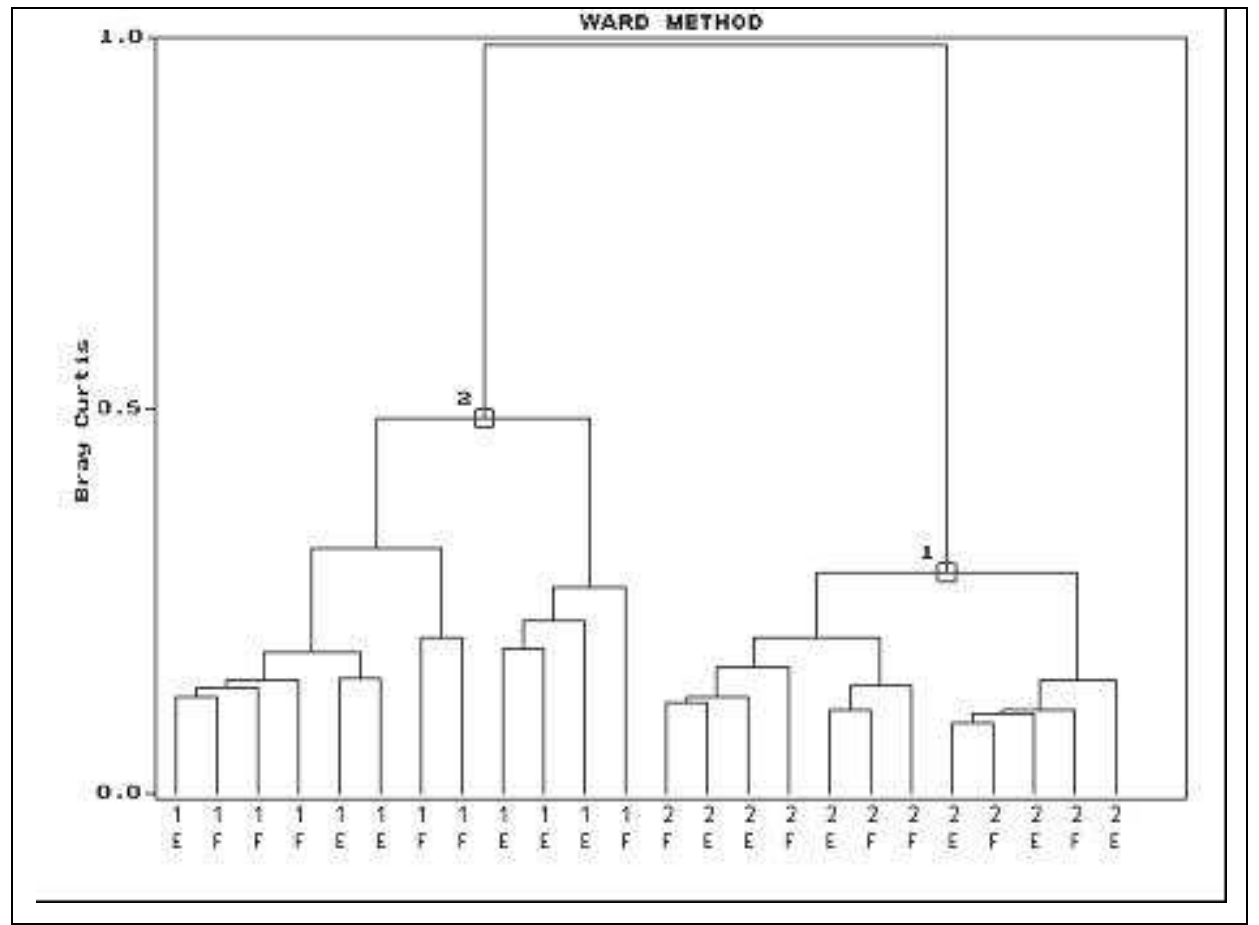

Figure 5 - Cluster analysis showing the sample groups at the continental shelf off Sergipe. Stations ( 1 and 2 ) and tide stages (E: ebb tide; F: flood tide).

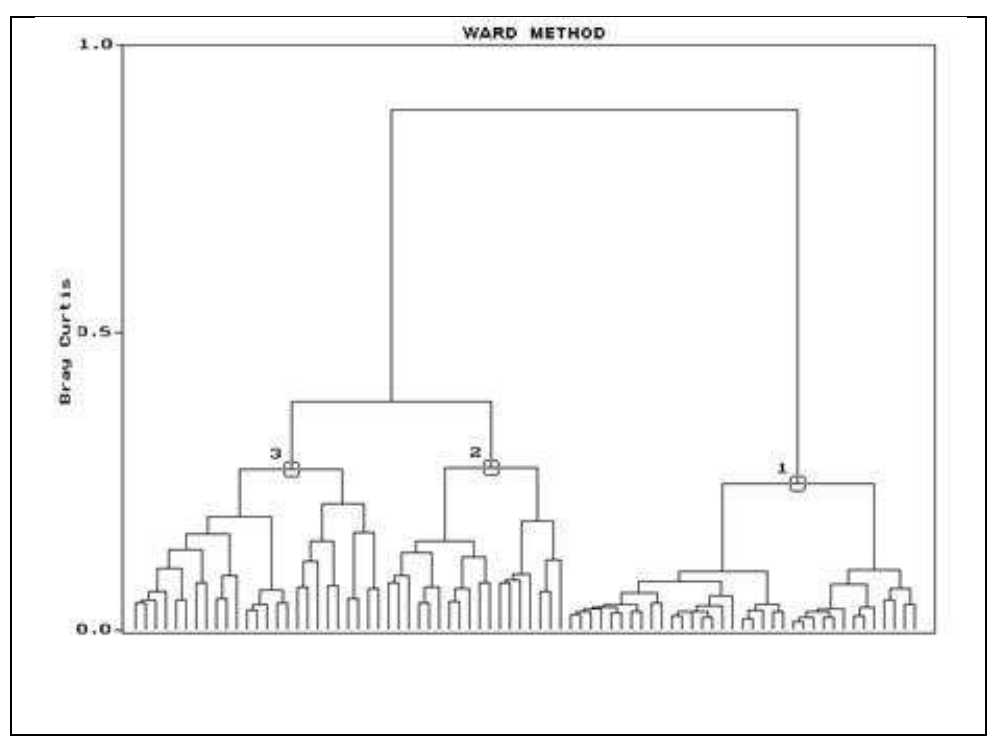

Figure 6 - Cluster analysis showing groups of taxa. The composition of each group is in table 3. 
ARAUJO; RIBEIRO. Distribution of the Mesozooplankton at continental shelf off Sergipe, Brazil.

Table 3 - Groups of taxa formed by the cluster analysis in December 2002 at the two stations in the continental shelf off Sergipe.

\begin{tabular}{|c|c|}
\hline GROUPS & TAXA \\
\hline 1 & $\begin{array}{l}\text { Aglaura hemistoma, Hydromedusae, Siphonophorae, Paracalanus } \\
\text { quasimodo, Paracalanus indicus, Paracalanus aculeatus, Paracalanus spp., } \\
\text { Calocalanus spp., Clausocalanus furcatus, Centropages furcatus, Euchaeta } \\
\text { marina, Temora stylifera, Calanopia americana, Mecynocera clausi, Oithona } \\
\text { plumifera, Oithona spp., Corycaeus giesbrechti, Corycaeus spp., Oncaea } \\
\text { media, Copepoda (nauplii), Ostracoda, Lucifer faxoni, Decapoda (larvae), } \\
\text { Bivalvia (larvae), Creseis acicula, Pteropoda, Gastropoda (larvae), } \\
\text { Ectoprocta (larvae), Echinodermata (larvae), Sagitta tenuis, Sagitta } \\
\text { enflata, Sagitta spp., Oikopleura longicauda, Oikopleura spp., Krohnitta sp. }\end{array}$ \\
\hline 2 & $\begin{array}{l}\text { Foraminiferida, Radiollaria, Liriope tetraphylla, Cnidaria, Polychaeta } \\
\text { (larvae), Nanocalanus minor, Undinula vulgaris, Acrocalanus longicornis, } \\
\text { Clausocalanus spp., Acartia danae, Calanidae, Farranula gracilis, Corycaeus } \\
\text { speciosus, Cirripedia (nauplii), Sagitta hispida, Salpida, Doliollida, Fritillaria } \\
\text { sp., }\end{array}$ \\
\hline 3 & $\begin{array}{l}\text { Subeucalanus pileatus, Calocalanus pavo, Parvocalanus crassirostris, } \\
\text { Candacia pachydactyla, Temora turbinata, Pontellopsis brevis, Labidocera } \\
\text { fluviatilis, Acartia lilljeborgi, Oithona tenuis, Oithona robusta, Oithona } \\
\text { setigera, Oithona oculata, Oithona nana, Euterpina acutifrons, } \\
\text { Harpacticoida, Corycaeus amazonicus, Oncaea venusta, Oncaea spp., } \\
\text { Copilia mirabilis, Cirripedia (cypris), Amphipoda, Isopoda, Oikopleura } \\
\text { dioica, Teleostei (eggs), Teleostei (larvae), }\end{array}$ \\
\hline
\end{tabular}

The water transparency and local depth correlated significantly to the mesozooplanktonic community distribution $(p<0.05)$ according to the Monte Carlo permutation test of canonical correspondence analysis. The correlation coefficients were 0.90 and 0.82 , respectively. The variance explained by the measured environmental factors was $76.5 \%$.

As one can see in the CCA biplot of samples and environmental variables, there is a clear spatial variation. The samples from station 1 are plotted on the right side of the CCA diagram and are associated to more transparent waters and high values of depth, temperature, $\mathrm{pH}$, ammonia and surface salinity. Acrocalanus longicornis, Neocalanus gracilis, Subeucalanus crassus, Lucicutia ovalis, Paracandacia simplex and Rhincalanus cornutus occured exclusively in this station. The samples from station 2 are plotted on the left side and nitrite, nitrate, silicate values were higher than at station 1 and surface salinity were lower (Fig. 7). Parvocalanus crassirostris, Temora turbinata, Acartia lilljeborgi, Oithona oculata, O. setigera, Euterpina acutifrons and Corycaeus amazonicus were the coastal and neritic species that occurred only at this station. 


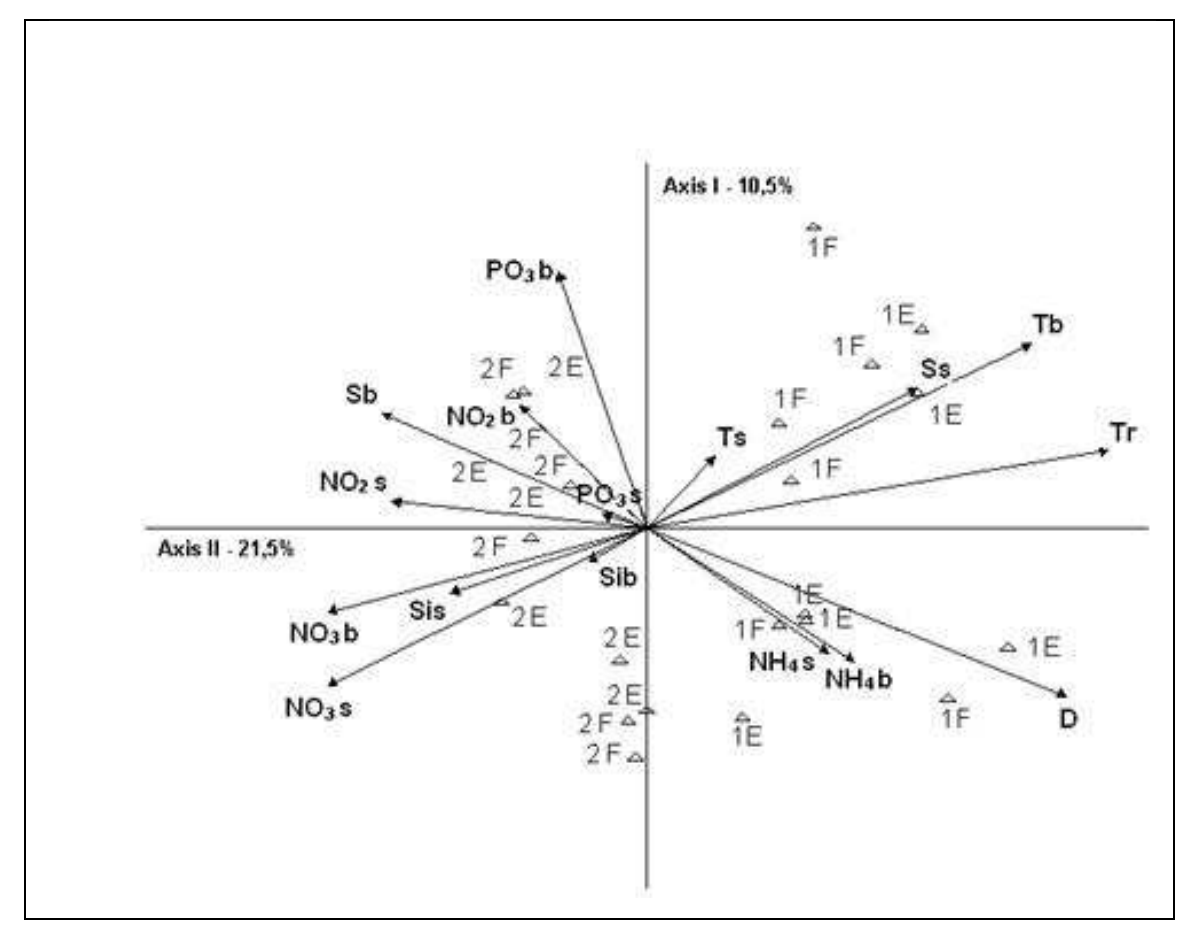

Figure 7 - Diagram of the first factorial canonic plan with samples and abiotic variables coordinates. Depth (D); temperature $(\mathrm{T})$; transparence $(\mathrm{Tr})$; salinity $(\mathrm{S})$; silicate (Si); surface (s); bottom (b); ebb tide (E); flood tide (F).

\section{DISCUSSION}

The mesozooplankton assemblage was characterized by coastal, neritic and oceanic tropical species. Diversity values were high and similar to those registered by NeumannLeitão et al. (1999) in the offshore-shelf region of the Brazilian Northeast.

Copepoda was the dominant taxon and contained the most frequent and abundant species of group 1 of the cluster analysis. Paracalanus quasimodo, Temora stylifera, Siphonophora, Creseis acicula and Fritillaria sp. were the most abundant holoplanktonic taxa, whereas Decapoda and Gastropoda larvae composed a significant part of the mesozooplanktonic community, specially at station 2 .

Statistic tests performed on the whole data set revealed a clear difference between the two stations in terms of mesozooplankton composition and abundance. At station 2, located close at the mouth of the São Francisco River holoplanktonic species and meroplanktonic larvae presented higher abundance values than at station 1. Parvocalanus crassirostris, Acartia lilljeborgi, Euterpina acutifrons, Corycaeus amazonicus occurred at this station only and their presence suggested the influence of the São Francisco plume on the mesozooplankton composition. The waters were more turbid and surface salinity values were lower in this area. These species are associated to coastal waters (BJORNBERG, 1963; 1981) and are frequent and abundant in estuaries, lagoons and embayments of Brazil coastal regions (LOPES, 1994; ESKINAZE-SANT'ANNA and TUNDISI, 1996; SILVA et al., 2003; ARA, 2004).

Paracalanus quasimodo was dominant at station 2 reaching $26.1 \%$ of the total mesozooplankton. It is a neritic species frequently registered in Brazilian shelf waters (ARAUJO et al., 1990; NEUMANN-LEITÃO et al., 1999; LOPES; BRANDINI; GAETA, 1999; SARTORI; LOPES, 2000). It was also the most frequent and abundant species in the shelf off Ubatuba (VEGA-PÉREZ; HERNANDEZ, 1997).

Paracalanidae copepods present herbivorous feeding strategies (PAFFENHÖFER, 1984). Food conditions may be the most important parameter in the distribution pattern of the mainly herbivorous copepods, which are by far the most abundant and attract the 
ARAUJO; RIBEIRO. Distribution of the Mesozooplankton at continental shelf off Sergipe, Brazil.

carnivores, which feed on them (KOUWENBERG, 1994). Paracalanidae dominance was probably related to phytoplankton production, due to nutrient contribution from São Francisco River or from local upwellings. Paes (2004) observed that in the areas close to the São Francisco and Japaratuba submarine canyons, the distribution of temperature and salinity in the water column suggested the occurrence of local upwelling, mainly in December.

The outflow of the São Francisco River had an influence on the hydrological conditions at the Sergipe shelf. The water column presented haline stratification. Salinity average value at surface was $30.8 \pm 1.9$ and at the bottom layer was $35.0 \pm 0.3 \mathrm{PSU}$. This condition allowed the development of a coastal zooplanktonic fauna, including benthic larvae usually registered in estuarine areas.

Estuaries are important to the adjacent ocean because they export organisms to the inner shelf waters (SCHWAMBORN et al., 1999) and replenish inorganic components, mainly phosphorates, nitrogenates and silicates (ESKINAZI-LEÇA et al., 2004). In the coast area off Galicia meroplankton were located in the inshore stations in consequence of outflow of coastal water from the Rias (VALDES et al., 1990). The relation between nutrients and high densities of the mesozooplankton in this area was shown by the canonical correspondence analysis.

Besides the influence of estuarine waters, the mesozooplankton was influenced by oceanic water masses. Subeucalanus pileatus, Clausocalanus arcuicornis, Phaena spinifera, Haloptilus mucronatus, Pleuromama piseki, Centropages longicornis, Microsetella rosea and Copilia mirabilis were exclusive species from station 2 and other oceanic species were also identified. These copepods are typical of the open sea (BRADFORD-GRIEVE et al., 1999) and may indicate the effect of oceanic water intrusions over the shelf off Sergipe.

At station 1, with a local depth of $27 \mathrm{~m}$ and more transparent waters, the epipelagic Thecosomata Creseis acicula was the most abundant species. It is registered in neritic and oceanic areas from tropical and subtropical regions. It is cited for the Brazilian coast and has been associated to the Brazil Current (VAN DER SPOEL; DADON, 1999). In the Copepoda assemblage Temora stilyfera was the most important species and according to Bradford-Grieve et al. (1999) it is a typical species from tropical and sub-tropical areas. Oceanic copepod species such as Neocalanus gracilis, Acrocalanus longicornis, Subeucalanus crassus, Lucicutia ovalis, Paracandacia simplex, Rhincalanus cornutus, Macrosetella gracilis, Oncaea media, Sapphirina nigromaculata and Pterosagitta draco occurred only in this station which presented higher salinity values, specially at the bottom. These species ocurred in low abundances, and do not seem to influence the community dynamic. The mesozooplankton was formed by a mixture of neritic and oceanic species usually registered at the Brazilian shelf waters. The oceanic species founded in the two stations reflected the effect of ocenic water intrusions on the Sergipe shelf.

The shelf and oceanic areas of the Brazilian Northeast are affected by the Tropical (TW) and South Atlantic Central Waters (SACW) (STRAMMA; IKEDA; PETERSON, 1990). According to PAES (2004) the salinity and temperature profiles showed that ocenic waters spread on the Sergipe shelf probably because it is narrow and interrupted by two big submarine canyons.

Further studies on physical oceanography of the Sergipe shelf area are necessary for better understanding of the interelation between hydrodynamic and the zooplankton distribution, which seems complex due to the estuarine influence and the oceanic water intrusions.

\section{CONCLUSIONS}

- The mesozooplankton was characterized by coastal, shelf and oceanic tropical species and their distribution suggests the influence of the outflow of the São Francisco River and oceanic water intrusions on the shelf off Sergipe. 
- A total of one hundred and three taxa were identified and Copepoda was the dominant group with fifty one species.

- Creseis acicula, Oikopleura spp., Temora stylifera, Paracalanus quasimodo, P. indicus and Aglaura hemistoma were the most abundant taxa at station 1. Paracalanus quasimodo, Siphonophora, Paracalanus spp., Temora stylifera, Decapoda and Gastropoda larvae occurred with greater abundance at station 2, located close to the São Francisco River mouth.

- Acrocalanus longicornis, Neocalanus gracilis, Subeucalanus crassus, Lucicutia ovalis, Paracandacia simplex and Rhincalanus cornutus occured exclusively in station 1. Parvocalanus crassirostris, Temora turbinata, Acartia lilljeborgi, Oithona oculata, O. setigera, Euterpina acutifrons and Corycaeus amazonicus were the coastal and neritic species that occurred only at station 2 where phosphate, nitrite, nitrate, silicate values were higher and surface salinity were lower.

- Mesozooplankton abundance presented spatial variation with higher values at the station located close to the São Francisco River plume.

\section{ACKNOWLEDGEMENTS}

This work was supported by "Petróleo Brasileiro S. A. and Financiadora de Estudos e Projetos (FINEP)". Chemical analyses were realized in the "Laboratório de Química Ambiental, Universidade Federal de Sergipe" under the responsability of Dr. José do Patrocínio Hora Alves.

\section{REFERENCES}

APHA (American public Health Association). Standard methods for the examination of water and wastewater. 19th ed. Washington, DC, 1995.

ARA, K. Temporal variability and production of the planktonic copepod community in the Cananéia Lagoon Estuarine System. Zoological Studies,Taipei, v. 43, n. 2, p. 179-186, 2004.

ARASHKEVICH, E.; WASSMANN, P.; PASTERNAK, A.; RISER, C. W. Seasonal and spatial changes in biomass, structure and development progress of the zooplankton community in the Barents Sea. Journal of Marine Systems, Amsterdam, v. 38, p. 125-145, 2002.

ARAUJO, H. M. P.; DÓRIA, R. A.; REZENDE, M. H. S. G.; LEAL, M. L. S. O. Copepoda das águas da plataforma continental do Estado de Sergipe. Anais do IV Encontro Brasileiro de Plâncton, Recife, v. 4, p. 283-291, 1990.

BJÖRNBERG, T. K. S. On the marine free-living copepods off Brazil. Boletim do Instituto Oceanográfico, São Paulo, v. 13, n. 1, p. 3-142, 1963.

BJÖRNBERG, T. K. S. Copepoda, p. 587-679. In: D. BOLTOVSKOY (Ed.). Atlas del zooplancton del Atlántico Sudoccidental y métodos de trabajos con el zooplancton marino. Mar del Plata: INIDEP, 1981. 936p.

BRADFORD-GRIEVE, J. M.; MARHASEVA, E. L.; ROCHA, C. E. F.; ABIAHY, B. Copepoda, p. 869-1098. In: D. BOLTOVSKOY (Ed.). South Atlantic Zooplankton. Leiden: Backhuys, 1999. 1706p.

COUTINHO, P. N. Sedimentos carbonáticos da plataforma continental brasilera. Revista de Geoogia, Fortaleza, p. 65-73, 1995.

CUNHA, S. B.; GUERRA, A. J. T. Geomorfologia do Brasil. Rio de Janeiro: Bertrand Brasil, 2003. 388p.

DESSIER, A.; DONGUY, J. R. Planktonic copepods and environmental properties of the eastern equatorial Pacific: seasonal and spatial variations. Deep-Sea Research, Oxford, v. 32, n. 9, p. 1117-1133, 1985.

ESKINAZI-LEÇA, E.; KOENING, M. L.; SILVA-CUNHA, M. G. G. Estrutura e dinâmica da comunidade fitoplanctônica, p. 353-374. In: E. ESKINAZI-LEÇA; S. NEUMANN-LEITÃO; M. 
ARAUJO; RIBEIRO. Distribution of the Mesozooplankton at continental shelf off Sergipe, Brazil.

F. COSTA (Eds.). Oceanografia: um cenário tropical. Recife: Universidade Federal de Pernambuco, 2004. 761p.

ESKINAZI-SANT'ANNA, E. M.; TUNDISI, J. G. Zooplâncton do estuário do Pina (RecifePernambuco-Brasil): composição e distribuição temporal. Revista Brasileira de Oceanografia, v. 44, n. 1, p. 23-33, 1996.

FRANÇA, A. M. C.; COUTINHO, P. N.; SUMMERHAYES, C. P. Sedimentos superficiais da margem continental nordeste brasileira. Revista Brasileira de Geologia, São Paulo, v. 6, n. 2, p. 71-88, 1976.

KOUWENBERG, J. H. M. Copepod distribution in relation to seasonal hydrographics and spatial structure in the North-western Mediterranean (Golfe du Lion). Estuarine Coastal and Shelf Science, London, v. 38, p. 69-90, 1994.

LOPES, R. M. Zooplankton distribution in the Guaraú River Estuary (South-eastern Brazil). Estuarine Coastal and Shelf Science, London, v. 39, p. 287-302, 1994.

LOPES, R. M.; BRANDINI, F. P.; GAETA, S. A. Distribution patterns of epipelagic copepods off Rio de Janeiro (SE Brazil) in Summer 1991/19992 and winter 1992. Hydrobiologia, Dordrecht, v. 411, p. 161-174, 1999.

NEUMANN-LEITÃO, S.; GUSMÃO, L. M. O.; SILVA, T. A.; NASCIMENTO-VIEIRA, D. A.; SILVA, A. P. Mesozooplankton biomass and diversity in coastal and oceanic waters off North-Eastern Brazil. Archieves of Fishery and Marine Research, Nehren, v. 47, n. 23, p. 153-165, 1999.

PAFFENHÖFER, G. A. Food ingestion by the marine planktonic copepod Paracalanus in relation to abundance and

size distribution of food. Marine Biology, New York, v. 80, p. 323-333, 1984.

PALOMARES-GARCÍA, R.; GÓMEZ-GUTIÉRREZ, J. Copepod community structure at Bahia Magdalena, Mexico during El Niño 1983-84. Estuarine Coastal and Shelf Science, London, v. 43, p. 583-595, 1996.

PARANAGUÁ, M. N.; NASCIMENTO-VIEIRA, D. A.; GUSMÃO, L. M. O.; NEUMANN-LEITÃO, S.; SCHWAMBORN, R. Estrutura da comunidade zooplanctônica, p. 441-458. In: E. ESKINAZI-LEÇA; S. NEUMANN-LEITÃO; M. F. COSTA (Eds.). Oceanografia: um cenário tropical.. Recife: Universidade Federal de Pernambuco, 2004. 761p.

PAES, E. T. Integração dos dados, p 705-721. In: Araujo, H. M. P. (Ed.).

Monitoramento da plataforma continental dos Estados de Sergipe e Alagoas sob influência de atividades petrolíferas. Aracaju: Fundação de Apoio à Pesquisa e Extensão de Sergipe/Universidade Federal de Sergipe, Relatório Técnico, v. II, 2004. 764 p.

PIELOU, E. C. Mathematical ecology. New York: Wiley-Intercience, 1977. 385p.

PINCA, S.; DALLOT, S. Zooplankton community structure in the Western Mediterranean sea related to mesoscale hydrodynamics. Hydrobiologia, Dordrecht, v. 356, p. 127-142, 1997.

RIOS-JARA, E. Spatial and temporal variations in the zooplankton community of Phosphorescent Bay, Puerto Rico. Estuarine and Coastal Marine Science, London, v. 46, p. 797-809, 1998.

SARTORI, L. P.; LOPES, R. M. Seasonal variability of pelagic copepod assemblages on the inner continental shelf off Paraná, Brazil. Nauplius, Botucatu, v. 8, n. 1, p. 79-88, 2000.

SCHWAMBORN, R.; EKAU, W.; SILVA, A. P.; SILVA, T. A.; SAINT-PAUL, U. The contribution of estuarine decapod larvae to marine macrozooplankton communities in Northeast Brazil. Archive of Fishery and Marine Research, Jena, v. 47, p. 201-210, 1999.

SHANNON, C. E.; WIENNER, W. A mathematical theory of communication. Bell System Technical Journal, San Diego, v. 27, p. 379-423, 1948. 
SILVA, T. A.; NEUMANN-LEITÃO, S.; SCHWAMBORN, R.; GUSMÃO, L. M. O.; NASCIMENTO-VIEIRA, D. A. Diel and seasonal changes in the macrozooplankton community of a tropical estuary in Northeastern Brazil. Revista Brasileira de Zoologia, Curitiba, v. 20, n. 3, p. 439-446, 2003.

STRAMMA, L.; IKEDA, Y.; PETERSON, R. G. Geostrophic transport in the Brazil Current region north of 200 S. Deep-Sea Research, Oxford, v. 37, p. 1875-1886, 1990.

STRICKLAND, J. D. H.; PARSONS, T. R. A. A manual of seawater analysis. Bulletin Fisheries Research Board of Canada, Ottawa, v. 125, p. 1-205, 1965.

SUMMERHAYES, C. P.; FAINSTEIN, R.; ELLIS, J. P. Continental margin off Sergipe and Alagoas northeastern Brazil: a reconnaissance geophysical study of morphology and structure. Marine Geology, Amsterdam, v. 20, p. 345-361, 1976.

TER BRAAK, C. J. F. Canonical correspondence analysis: a new eigenvector technique for multivariate direct gradient analysis. Ecology, Washington, v. 67, p. 1167-1179, 1986.

TER BRAAK, C. J. F. The analysis of vegetation-environment relationship by canonical correspondence analysis. Vegetatio, Dordrecht, v. 69, p. 69-77, 1987.

TER BRAAK, C. J. F. CANOCO - an extension of DECORANA to analysis speciesenvironment relationships. Hydrobiologia, Dordrecht, v. 184, p. 169-170, 1989.

VAldes, J. L.; Roman, M. R.; Alvarez-Ossorio, M. T.; Gauzens, A. L.; miranda, A. Zooplankton composition and distribution off the coast of Galicia, Spain. Journal of Plankton Research, Oxford, v. 12, n. 3, p. 629-643, 1990.

VAN DER SPOEL, S.; DADON, J. R. Pteropoda, 649-706. In: D. Boltovskoy (Ed.). South Atlantic Zooplankton. Leiden: Backhuys, 1999. 1706 p.

VEGA-PÉREZ, L. A.; HERNANDEZ, S. Composição e distribuição da Família Paracalanidae (Copepoda: Calanoida) ao largo de São Sebastião, Estado de São Paulo-Brasil, com ênfase em três espécies de Paracalanus. Revista Brasileira de Oceanografia, São Paulo, v. 45, n. 1/2, p. 61-75, 1997.

VILLWOCK, J. A. A costa brasileira: geologia e evolução. Notas Técnicas, Porto Alegre, CECO/IG/UFRGS, v. 7, p. 38-49, 1994.

WIAF, G.; FRID, C. L. J. Short-term temporal variation in coastal zooplankton communities: the relative importance of physical and biological mechanisms. Journal of Plankton Research, Oxford, v. 18; n. 8, p. 1485-1501, 1996.

ZAR, J. H. Bioestatistical Analysis. New Jersey: Prentice-Hall, 1984. 620p. 